Bull. Austral. Math. Soc.

$20 \mathrm{H} 20,20 \mathrm{C} 15,20 \mathrm{G} 40$

VOL. $62(2000) \quad[335-345]$

\title{
MINIMALLY IRREDUCIBLE GROUPS OF PRIME DEGREE
}

\author{
F. Dalla Volta and L. Di Martino
}

We determine the irreducible subgroups $G$ of $G L(r, \mathbb{C})$, where $r$ is prime and all proper subgroups of $G$ are reducible.

\section{INTRODUCTION}

A linear group is said to be minimally irreducible if it is irreducible, and all its proper subgroups are reducible. It was shown by Platonov (see [10]) that a minimally irreducible subgroup of $G L(n, \mathbb{C})$ is necessarily finite. A certain number of papers on minimally irreducible groups have appeared over the years, mostly by Russian authors and mostly focused on soluble groups. In this paper, we determine the minimally irreducible subgroups $G$ of $G L(n, \mathbb{C})$, when the degree $n$ is a prime. The case when $G$ is a soluble group was dealt with by D.A. Suprunenko in the early 1970's (see Section 2). In dealing with the non-soluble case, we rely heavily on recent papers on linear groups of prime degree by Dixon and Zalesskii [3, 4]. The analysis is reduced, exploiting the minimality assumption, to quasi-simple groups (Section 3), and even to simple ones if $G$ is primitive (see 3.3). Interesting occurrences are the monomial representations of $P S L(n, q)$ of degree $r=\left(q^{n}-1\right) /(q-1)$, and the Weil representations of the unitary groups $P S U(n, q)$ of degree $r=\left(q^{n}+1\right) /(q+1)$. The final result is summarised in Theorem 1 , at the end of Section 3.

\section{The SOluble CASE}

The soluble minimally irreducible groups of prime degree have been determined and explicitly described up to conjugacy by Suprunenko (see $[14,15]$ ). The key result leading to their classification is the following lemma, of which we provide a proof, for the reader's convenience.

LEMmA 1. Let $G$ be a minimally irreducible subgroup of $G L(r, \mathbb{C}), r$ a prime, and assume that $G$ is a soluble group. Then $G$ is a (non-Abelian) group all of whose subgroups are Abelian.

Received 17th April, 2000

Copyright Clearance Centre, Inc. Serial-fee code: 0004-9727/00 \$A2.00+0.00. 
Proof: Let $N$ be a maximal normal subgroup of $G$. As $N$ is reducible, by Clifford's theorem the irreducible constituents of $N$ all have degree 1. Hence $N$ is Abelian and $|G: N|=r$. (In fact, $N=S_{1} \times \cdots \times S_{t} \times P_{1}$, where each $S_{i}$ is an (Abelian) Sylow $q_{i}$-subgroup of $G, q_{i} \neq r$, and $P_{1}$ has index $r$ in a Sylow $r$-subgroup of $G$.) Now, let $H$ be any proper subgroup of $G$ not contained in $N$. Then $H \cap N$ is a normal Abelian subgroup of $H$ of index $r$. By Ito's theorem, any irreducible representation of $H$ has degree 1 or $r$. As $H$ is a reducible subgroup of $G$, it follows that $H$ is Abelian.

The groups satisfying the conditions stated in the Lemma are known as MillerMoreno groups. They have been classified by Rédei in 1947 (see [11]). In principle, those admitting a minimally irreducible representation of prime degree can be read off from there. However, Suprunenko's analysis in the quoted papers is direct, and does not exploit nor quote [11]. For our purposes in this paper it will be enough to record the following facts:

Proposition 1. $\quad[14,15]$ Let $G$ be a minimally irreducible soluble subgroup of $G L(r, \mathbb{C}), r$ a prime. Then either

(1) $G$ is an $r$-group; or

(2) $G$ is the semidirect product of an elementary Abelian $u$-group of order $u^{l}$ by a cyclic $r$-group, where $l$ is the least integer such that $r \mid u^{l}-1$. In particular, $G$ is a monomial group.

\section{The non-soluble Case}

3.1. Reduction to The quasi-Simple CASE The following Proposition drastically reduces the analysis of minimally irreducible non-soluble linear groups of prime degree.

PRoposition 2. Let $G$ be a minimally irreducible subgroup of $G L(r, \mathbb{C}), r$ a prime, and assume that $G$ is non-soluble. Then $G$ is quasi-simple.

ProOF: Let $N$ be a maximal normal subgroup of $G$. By Clifford's theorem, either $N$ has a single homogeneous component on $V(r, \mathbb{C})$, in which case $N$ is scalar and we are done, or $N$ splits into $r$ distinct irreducible constituents of degree 1, and these describe all the irreducible $N$-submodules of $V(r, \mathbb{C})$. Suppose the latter occurs. Then, we claim that there exists $x \in G-N$ such that $\langle N, x\rangle$ is irreducible, and therefore $\langle N, x\rangle=G$. Indeed, as $G$ acts transitively on the irreducible constituents of $N$ (alternatively: by Ito's theorem, as in the present instance $N$ is Abelian), $r$ divides $|G: N|$. Thus, we may choose $x \in G-N$ such that $x$ acts as a cycle of length $r$ on the constituents of $N$, and as such, does not preserve any partial sum of these constituents. It follows that $\langle N, x\rangle$ acts irreducibly on $V(r, \mathbb{C})$, whence $\langle N, x\rangle=G$. Since $x^{r} \in N,|G: N|=r$, and we may proceed exactly as in the soluble case, concluding that all proper subgroups of $G$ are Abelian. In particular, $G$ must be soluble, a contradiction. 
At this stage, we distinguish two cases: (1) $G$ acts imprimitively on $V(r, \mathbb{C})$, that is $G$ is a group of monomial matrices; (2) $G$ acts primitively on $V(r, \mathbb{C})$.

3.2. The monomial case In order to deal with this case, we need to recall the list of 2-transitive simple permutation groups of prime degree $r$ (a well-known consequence of the classification of finite simple groups). These are: the alternating groups $\mathbb{A}_{r}, r \geqslant 5$, in their natural action; the projective special linear groups $P S L(n, q), n>1$, with $r=\left(q^{n}-1\right) /(q-1)$ (acting on the points of the projective space $P G(n-1, q)$; the group $P S L(2,11)$, with $r=11$; the Mathieu group $M_{11}$, with $p=11$; the Mathieu group $M_{23}$, with $r=23$.

Then the following result gives the desired answer. Though it is essentially embodied in [4], it is convenient for our purposes to give here a detailed proof.

PRoposition 3. Let $G$ be a quasi-simple finite subgroup of $G L(r, \mathbb{C}), r$ a prime, and assume that $G$ acts monomially and irreducibly on $V(r, \mathbb{C})$. Then $G$ is one of the following:
(a) $\mathbb{A}_{5}, r=5$;
(b) $P S L(n, q)$, with $n \geqslant 2, q>2$, and $r=\left(q^{n}-1\right) /(q-1)$;
(c) $\operatorname{PSL}(3,2), r=7$;
(d) $M_{11}, r=11$.

Proof: By assumption $G$ is monomial: in other words $G$ is conjugate to a subgroup of the semidirect product $M=\left[D_{r}\right] \mathbb{S}_{r}$, where $\mathbb{S}_{r}$ denotes the subgroup of $G L(r, C)$ consisting of the permutation matrices, and $D_{r}$ denotes the subgroup of $G L(r, C)$ consisting of the diagonal matrices of finite order. Moreover, since $G$ is quasi-simple, $G \subseteq S L(r, C)$, and $Z(G)$ is the trivial group or has order $r$. Let $\gamma$ be the restriction to $G$ of the natural epimorphism $M \rightarrow \mathbb{S}_{r}$, and set $H=\gamma(G)$. Clearly, $\operatorname{ker}(\gamma)=D_{r} \cap G=Z(G)$. It is shown in [4, Lemma 3.1] that $G$ splits over $\operatorname{ker}(\gamma)$, provided $r>2$ (which is our case, since $G$ is supposed to be quasi-simple). Therefore $\operatorname{ker}(\gamma)=1$, and $G \simeq H$ is simple. Now, by a classical theorem of Burnside, $H$ is a 2-transitive permutation group of degree $r$. Hence $G$ is isomorphic to one of the groups listed above. The alternating groups $\mathbf{A}_{r}, r>5$, are immediately ruled out. Indeed, a point-stabiliser in $\mathbf{A}_{r}$ is isomorphic to $\mathbf{A}_{r-1}$, which is itself simple. Therefore the only monomial representation of degree $r$ of $\mathbf{A}_{r}$ is the natural permutation representation, which is obviously reducible. (In fact, it can be easily shown that $\mathbf{A}_{r}$ has no irreducible representation of degree $r$ whatsoever.) On the other hand, $\mathbf{A}_{4}$ has two non-trivial linear representations, which induce one and the same monomial irreducible representation of $\mathbf{A}_{5}$ of degree $5 . M_{23}$ is also ruled out, since it has no irreducible representation of degree 23. $P S L(2,11)$ does have a unique irreducible representation of degree 11 . However this representation is not monomial, as the only subgroup of $\operatorname{PSL}(2,11)$ of index 11 is isomorphic to $\mathbf{A}_{5}$. As for $M_{11}$, it has a 
unique irreducible representation of degree 11 , which is indeed monomial, induced from a non-trivial linear representation of $M_{10}$. Thus, we are left with the case $G=P S L(n, q)$. Here the candidates are the representations induced from the non-trivial linear representations of the stabiliser $P_{1}$ of a point of $P G(n-1, q)$. Since $\left|P_{1}: P_{1}^{\prime}\right|=q-1$, if $q>2$ the required representations do exist, and are known to be irreducible (for example, see [18, Proposition 1.9]). If $q=2,\left|P_{1}: P_{1}^{\prime}\right|=1$, unless $n=3$, in which case $\left|P_{1}: P_{1}^{\prime}\right|=2$. The group $P S L(3,2)$ does have a unique irreducible representation of degree 7 , which is monomial.

\section{REMARKS.}

1. Note that the point-stabilisers are the only subgroups of index $\left(q^{n}-1\right) /(q-1)$ of $\operatorname{PSL}(n, q)$. Indeed: (i) any such subgroup is maximal and contains a Sylow $p$-subgroup $\left(q=p^{a}\right.$ ); (ii) any subgroup of a simple Chevalley group satisfying (a) must contain a Borel subgroup, hence is parabolic; whence our contention, by order comparison. Also, note that $r=\left(q^{n}-1\right) /(q-1)$ can be a prime only if $n$ is a prime, $q=p^{n^{k}}$, and $(n, q-1)=1$.

2. The representation of degree 5 of $A_{5}$ is minimally irreducible, and also appears in (b), for $n=2, q=4$.

3. The representation of degree 7 of $\operatorname{PSL}(3,2)$ is also minimally irreducible, since the only subgroup of order divisible by 7 is $\left[C_{7}\right] C_{3}$.

4. Let $\sigma$ denote the aforementioned representation of degree 11 of $M_{11}$. Then $\sigma$ is minimally irreducible. For, assume the contrary. Since a maximal subgroup $H$ of $M_{11}$ having order divisible by 11 is necessarily isomorphic to $\operatorname{PSL}(2,11)$, it follows that $\left.\sigma\right|_{P S L(2,11)}$ must be irreducible and monomial. This cannot occur, as observed in the proof of the Proposition above.

Next, we prove:

PROPOSITION 4. Let $\chi_{P}$ be one of the monomial irreducible representations of the group $G=P S L(n, q)$ of degree $r=\left(q^{n}-1\right) /(q-1)$. Then $\chi_{P}(G)$ is minimally irreducible.

Proof: Suppose the contrary. Then there is a proper subgroup $K$ of $G$ such that $\chi_{P}(K)$ is irreducible. Clearly $\chi_{P}(K)$ is monomial, and without loss of generality, we may assume that $\chi_{P}(K)$ is minimally irreducible. It follows that either $K$ is soluble, or $K$ is quasi-simple. In the latter case, $K$ must be one of the groups listed in Proposition 3 above. $K=P S L(3,2), r=7$ cannot occur, since it would force $n=3, q=2$, whence $K=G . \quad r=11$ is incompatible with the condition $r=\left(q^{n}-1\right) /(q-1)$, and this rules out $K=M_{11}$. Thus we are left with the possibility of an irreducible embedding $K=P S L(m, t)<P S L(n, q)$, where $r=\left(t^{m}-1\right) /(t-1)=\left(q^{n}-1\right) /(q-1)$. We proceed to show that this cannot occur. First, we note that, since the embedding is proper, the condition $(*)\left(t^{m}-1\right) /(t-1)=\left(q^{n}-1\right) /(q-1)$ forces $(t, q)=1$. Thus, the 
embedding can be interpreted as an irreducible projective representation of the group $\operatorname{PSL}(m, t)$ in cross-characteristic, and therefore by [9] we obtain the bound $n \geqslant t^{m-1}-1$. From (*) we get $t\left(t^{m-2}+\cdots+t+1\right)=q\left(q^{n-2}+\cdots+q+1\right)$, or in other words $t \cdot\left(t^{m-1}-1\right) /(t-1)=q \cdot\left(q^{n-1}-1\right) /(q-1)$. Whence $q \cdot\left(q^{n-1}-1\right) /(q-1) \leqslant t \cdot n /(t-1)$. This in turn implies $q(t-1) / t \cdot\left[\left(q^{n-1}-1\right) /(q-1)\right] \leqslant n$. Since $q(t-1) / t \geqslant 1$, it follows $\left(q^{n-1}-1\right) /(q-1) \leqslant n$, a contradiction unless $n=2$. But the latter cannot be, since it would imply $K=P S L(2,2)$ or $P S L(2,3)$, which is not the case.

Now assume that $K$ is soluble. We know that either $K$ is an $r$-group, or $K \simeq\left[E_{u^{l}}\right] C_{r^{\text {s }}}$ ( $u$ a prime and $l$ the least integer such that $u^{l} \equiv 1(r)$ ). Since $r=\left(q^{n}-1\right) /(q-1)$ divides $|G|$ at the first power, the former case cannot occur, and in the latter case $s=1$. Denote by $\widetilde{K}$ and $\langle\widetilde{x}\rangle$, respectively, the pre-images of $K$ and $C_{r}$ in $S L(n, q)$. Then $\widetilde{x}$ is a socalled 'Singer-cycle', acting fixed-point freely on $V(n, q)$. In particular, $\langle\widetilde{x}\rangle$ is a maximal irreducible and primitive cyclic subgroup of $S L(n, q)$. Now it follows from the knowledge of maximal irreducible soluble subgroups of $S L(n, q)$ (for example, see [13, p.162], taking into account the condition $(n, q-1)=1)$, that $\widetilde{K}$ must be contained in the normaliser of $\langle\widetilde{x}\rangle$. But this would force $K$ to be Abelian, a contradiction.

3.3. The PRIMITIVe CASE In the primitive case, we obtain from the start a further reduction, namely:

PROPOSITION 5. If $G$ is a primitive minimally irreducible quasi-simple subgroup of $G L(r, \mathbb{C})$, then $G$ is a simple group.

Proof: As already observed, the assumption that $G$ is quasi-simple implies that $G$ is a subgroup of $S L(r, \mathbb{C})$ and $Z(G)$ is the trivial group or has order $r$. Let $1 \neq R$ be an $r$-Sylow subgroup of $G$. We claim that $R$ is necessarily Abelian. Indeed, by our assumptions, $R$ is reducible, and hence decomposes into $r$ irreducible constituents of degree 1 . But this obviously implies that $R$ is Abelian, as claimed. Now, we may appeal to $[1$, Theorem $4 \mathrm{~A}]$, where it is proved that $G$ splits over $Z(G)$. This is a contradiction, since $G$ is perfect.

The above reduction allows us to make direct use of the list of groups appearing in [3, Theorem 1.2]. Namely, we may exploit the following:

Result. $[9,3]$ Let $G$ be a finite primitive simple subgroup of $S L(r, \mathbb{C})$. Then one of the following cases occur:

(1) $G \simeq \mathbf{A}_{r+1}, r \geqslant 7$.

(2) $G \simeq P S L(2, q)$, with $r$ and $q=p^{a}$ satisfying one of the following conditions:

(i) $r=p=q \geqslant 11$,

(ii) $r=(q+1) / 2, q=p^{2^{k}} \geqslant 5$ for some odd prime $p$ and $k \geqslant 0$,

(iii) $r=(q-1) / 2, q$ a prime or $q=3^{a}$ for some odd prime $a$, 
(iv) $r=q-1, q=2^{a}$ for some odd prime $a$.

(3) $G \simeq P S p(2 n, q)$, with $r, n$ and $q=p^{a}$ satisfying one of the following conditions:

(i) $r=\left(q^{n}+1\right) / 2$, where $n>1$ is a power of 2 , and $q=p^{2^{k}}$ for some odd prime $p$ and $k \geqslant 0$,

(ii) $r=\left(3^{n}-1\right) / 2$, where $q=3$ and $n$ is an odd prime.

(4) $G \simeq P S U(n, q), r=\left(q^{n}+1\right) /(q+1), n$ an odd prime.

(5) $G \simeq P S p(6,2), r=7$.

(6) $G \simeq M_{12}, r=11$.

(7) $\mathrm{G} \simeq \mathrm{Co}_{2}, \mathrm{Co}_{3}$ or $\mathrm{M}_{24}, r=23$.

In order to finish off, we shall carry out a case by case analysis, determining which of the groups and representations listed above are minimally irreducible, and which are not.

\section{The alternating groups}

The alternating group $\mathbb{A}_{5}$ has two irreducible representations of degree 3 , which are not minimally irreducible, as their restrictions to $\mathbb{A}_{4}$ are irreducible $\left(\mathbb{A}_{5}\right.$ also has a unique irreducible representation of degree 5 , and this is monomial, as noted above). The alternating group $\mathbb{A}_{6}$ has two irreducible representations of degre 5 , which are not minimally irreducible, as their restrictions to $\mathbb{A}_{5}$ are irreducible. The group $\mathbb{A}_{7}$ has no irreducible representations of prime degree. The general case is dealt with by the following:

PRoposition 6. For all $n>7$, the alternating group $\mathbf{A}_{n}$ is not minimally irreducible of prime degree $r$.

PROOF: It is known that if $n>7$ then $\mathbb{A}_{n}$ has an irreducible representation of prime degree $r$ if and only if $n=r+1$ (for example, see [3]). Such representation is unique, and appears as the non-trivial constituent $\chi$ of the natural permutation representation $\psi$ of $\mathbf{A}_{r+1}$. Without loss of generality, we may view $\mathbf{A}_{r+1}$ as acting on the $r+1$ points of the projective line $P G(1, r)$. Clearly, the projective group $P S L(2, r)$ is a subgroup of $\mathbf{A}_{r+1}$, and $\left.\psi\right|_{P S L(2, r)}=1+\left.\chi\right|_{P S L(2, r)}$ realises the 2-transitive action of $P S L(2, r)$ on the points of $P G(1, r)$. Hence $\left.\chi\right|_{P S L(2, r)}$ is irreducible, and we are done.

\section{2. $G \simeq P S L(2, q)$}

Let $\psi$ denote a complex irreducible representation of $G$ of degree $r$.

(i) $r=p=q$.

Assume that $\psi$ is not minimally irreducible. Then there exists a maximal subgroup $H$ of $G$, such that $p$ divides $|H|$ and $\psi_{\mid H}$ is irreducible. The list of maximal subgroups of $P S L(2, p)$ (for example, see [7]) gives us as candidates for $H$ only the $p$-Sylow normalisers. 
These have order $p(p-1) / d$, where $d=(2, p-1)$, and by Ito's theorem $r=p$ should divide $(p-1) / d$, a contradiction.

(ii) $r=(q+1) / 2$.

Assume that $\psi$ is not minimally irreducible, and argue as in (i). Scrutiny of the list of maximal subgroups rules out all candidates for $H$, thus giving a contradiction, except for $q=5, r=3$ and $q=9, r=5$.

(iii) $r=(q-1) / 2$.

Look at $\psi_{\mid H}$, where $H$ is the normaliser of a Sylow $p$-subgroup $P$ of $G$. The subgroup $P$ is elementary Abelian of order $q$, and $|H|=q(q-1) / 2=q r$. Again by Ito's theorem, an irreducible representation of $H$ has either degree 1 or degree $r$. Thus $\psi_{\mid H}$ is irreducible, for otherwise it would split into $r$ constituents of degree 1 , and therefore $H$ would be Abelian, which is not the case whenever $q>3$. We conclude that $\psi$ is not minimally irreducible.

(iv) $r=2^{s}-1, q=2^{s}$.

Same argument as in (iii). $\psi$ is not minimally irreducible.

3. The symplectic groups

The representations of $P S p(2 n, q)$ in question are the so-called Weil representations, with the constraints on $n$ and $q$ arising from the assumption that $r$ must be a prime. Namely, $\bar{G}=S p(2 n, q), q$ odd, has exactly two irreducible representations $\xi, \xi^{*}$ of degree $x=\left(q^{n}-1\right) / 2$, and exactly two irreducible representations $\eta, \eta^{*}$ of degree $x+1=$ $\left(q^{n}+1\right) / 2$ over the complex field. These are commonly referred to as the Weil representations of the symplectic group, and have been studied by several authors. Their construction, as well as the description of some basic properties, can be found in $[17,6,12,5]$, while their uniqueness has been proved in [16]. In particular, by [9] $\xi$ and $\xi^{*}$ are the unique irreducible complex representations of $\bar{G}$ of minimal degree $>1$. It is also known (see [16, Lemma 2.6], that the central involution $-I$ lies in the kernel of $\xi\left(\xi^{*}\right)$ if and only if $n$ is even, or $n$ is odd and $q \neq \equiv 1(\bmod 4)$, whereas $-I$ lies in the kernel of $\eta$ $\left(\eta^{*}\right)$ if and only if $n$ is even, or $n$ is odd and $q \equiv 1(\bmod 4)$. Thus, under these restrictions on $n$ and $q$, they actually provide representations of the group $G=P S p(2 n, q)$. For our purposes it is also useful to recall the following (for example, see [6]): for any $g \in S p(2 n, q)$, denoting by $c(g)$ the dimension of the subspace of $V(2 n, q)$ fixed pointwise by $g,|(\xi+\eta)(g)|^{2}=q^{c(g)}$. Moreover, if $g$ has order coprime to $q,(\xi+\eta)(g)(\xi+\eta)(g)$ is a rational integer. Similarly for the conjugate representation $\xi^{*}+\eta^{*}$.

Proposition 7. The irreducible Weil representations of $P S p(2 n, q)$ are not minimally irreducible.

ProOF: It is well-known that there are standard embeddings $S p\left(2 m, q^{r}\right) \hookrightarrow S p(2 n, q)$, $n=m r, r>1$ (for example, see [7, p.228]). As for the representations $\xi, \xi^{*}$, it was no- 
ticed in [12] that $\xi$ and $\xi^{*}$ are still irreducible when restricted to $S p\left(2 m, q^{r}\right)$ [this is simply because the minimal degree of a non-linear irreducible representation is the same for the two groups, namely $\left.\left(q^{m r}-1\right) / 2\right]$. Thus we are left to deal with the case (3), (i): without loss of generality, the relevant representation may be chosen to be $\eta$. Let us consider in particular the embedding $S L\left(2, q^{n}\right) \simeq S p\left(2, q^{n}\right) \hookrightarrow S p(2 n, q)$. The subgroup $H=S L\left(2, q^{n}\right)$ has elements $h$ of order $q^{n}+1$, which, viewed as elements of $S p(2 n, q)$ correspond to 'Singer cycles' acting fixed-point freely on $V(2 n, q)$. Thus $|(\xi+\eta)(h)|=1(*)$. Now assume that $\eta_{\mid H}$ is reducible: then, by the minimality of $\xi_{\mid H}\left(\xi_{\mid H}^{*}\right), \eta_{\mid H}=1_{H}+\xi_{\mid H}$ (or $\xi_{\mid H}^{*}$ ), whence $(\xi+\eta)(h)=\xi(h)+1+\xi(h)$ (or $\xi^{*}(h)$ ). Since $h$ can be chosen such that $\xi(h)=\xi^{*}(h)=1$ (for example, see [2, Section 38], it follows $(\xi+\eta)(h)=3$, contradicting $(*)$.

\section{The unitary groups.}

As in the symplectic case, these representations of $P S U(n, q)$ are usually called Weil representations. Namely, $S U(n, q)$ has $q$ irreducible representations $\zeta^{i}(1 \leqslant i \leqslant q)$ of degree $\left(q^{n}-(-1)^{n}\right) /(q+1)$. They arise from the symplectic ones via the standard embedding $U(n, q) \hookrightarrow S p(2 n, q)$. For their construction, see for example, [12, 5], and especially [16, Section 4]. Finally observe that, since $n$ is an odd prime, and $r$ is a prime, necessarily $(n, q+1)=1$. Hence under our assumptions $S U(n, q) \simeq \operatorname{PSU}(n, q)$.

Proposition 8. Let $G=\operatorname{PSU}(n, q)$, and denote by $\varphi$ an irreducible Weil representation of $G$ of prime degree $r=\left(q^{n}+1\right) /(q+1)$, where $n$ an odd prime. Then $\varphi$ is minimally irreducible, except for $n=q=3$.

Proof: Suppose the contrary. Then $\phi_{\mid M}$ is irreducible for some maximal subgroup $M$ of $G$ (in particular, $r$ must divide $|M|$ ). We show that this cannot occur unless $n=q=3$, using 'brute force', namely we check out all possible cases, relying on the knowledge of maximal subgroups of $G$ (see [8], to which we refer for the description of the so-called 'Aschbacher classes', the 'exceptional' class $\mathcal{S}$ and all other details).

(1) $M$ belongs to one of the 'Aschbacher classes' $\mathcal{C}_{i}(1 \leqslant i \leqslant 7)$, as described in [8]. As $n$ is a prime, the classes $\mathcal{C}_{4}$ and $\mathcal{C}_{7}$ are ruled out from the very start.

Suppose that $M$ belongs to the class $\mathcal{C}_{1}$, that is $M$ is either a parabolic or the stabiliser of a non-degenerate subspace. Looking at the orders of the groups involved, we observe that, if $s$ is any prime divisor of $|M|$, then either $s=p$, or $s \mid\left(q^{i} \pm 1\right)$ for some $i<n$. However, $r=\left(q^{n}+1\right) /(q+1)$ does not divide $q^{i} \pm 1$ if $i<n$. Thus we are left with the classes $\mathcal{C}_{2}, \mathcal{C}_{3}, \mathcal{C}_{5}$ and $\mathcal{C}_{6}$. In the first case, $M$ is imprimitive on $V=V\left(n, q^{2}\right)$. Thus, since $n$ is a prime, $M$ is monomial, and again this possibility is ruled out, as $r$ does not divide $(q+1)^{n-1} \cdot n !$. The class $\mathcal{C}_{3}$ (groups over field extensions) contains subgroups of order divisible by $r$. These are precisely the $r$-locals of type $\left[C_{r}\right] C_{n}$, and are obviously reducible. In class $\mathcal{C}_{5}$ (subfield stabilisers), we only have to worry about 
subgroups of type $U\left(n, q_{0}\right), q=q_{0}^{a}$. But these cannot be irreducible, since this would force $\left(q^{n}+1\right) /(q+1)=\left(q_{0}^{n}+1\right) /\left(q_{0}+1\right)$. As for $\mathcal{C}_{6}$ (normalisers of $s$-groups of symplectic type, $\left.n=s^{m}\right)$, the assumption on $n$ forces $n=s$ and $M$ of type $n^{2} \cdot S p(2, n)$. But then $r$ does not divide $|M|$ (except for $n=3, q=2$ ), and this case is equally ruled out.

(2) $M$ belongs to the class $\mathcal{S}$. Thus $M$ is an almost simple group, acting projectively and absolutely irreducible on $V=V\left(n, q^{2}\right)$. Note that, under our assumptions, denoting by $S$ the simple socle of $M, \phi_{\mid S}$ is still irreducible. We observe the following:

(i) $S$ cannot be a classical group over a field of characteristic $p$ (as above, by order reasons: $r=\left(q^{n}+1\right) /(q+1)$ does not divide $q^{i} \pm 1$ if $\left.i<n\right)$.

(ii) Suppose that $S$ is a classical group over a field of characteristic coprime to $p$. Then, by Proposition 3 and [3], only the following cases may occur:

(a) $S=P S L(2, t)$, and either $\phi(1)=t, t$ prime $\geqslant 11$, or $\phi(1)=(t \pm 1) / 2$, or $\phi(1)=t-1$

(b) $S=P S L(m, t)$, and $\phi(1)=\left(t^{m}-1\right) /(t-1)$;

(c) $S=P S p(2 m, t)$, where $t$ is odd, and $\phi(1)=\left(t^{m} \pm 1\right) / 2$;

(d) $S=P S U(m, t)$, where $m$ is an odd prime, and $\phi(1)=\left(t^{m}+1\right) /(t+1)$;

(e) $S=P S p(6,2), \phi(1)=7$;

(f) $\operatorname{PSL}(3,2), \phi(1)=7$.

Each such $S$, under our assumptions, admits an absolutely irreducible projective representation on $V$. Thus, it must satisfy the lower bounds given in [9] (reproduced with a few amendments in $[8$, p.188, Table 5.3.A]) for the representations of groups of Lie type in cross-characteristic. Direct computation in cases (a) to (e) shows that this cannot occur, except for case (a) when $n=q=3$. The latter case is a true exception, since the representation of $\operatorname{PSU}(3,3)$ of degree 7 admits an irreducible restriction to $\operatorname{PSL}(2,7)$ (this also covers case (f)).

(iii) Suppose $S$ is alternating. Then, noting that $r \geqslant 7$, it follows from Proposition 3 and [3] that the only possible occurrence is $\mathbf{A}_{r+1}$. But $|P S U(n, q)|<\left|\mathbf{A}_{r+1}\right|$, and we are done.

(iv) $S$ cannot be a sporadic group. Indeed, again by Proposition 3 and [3], the only sporadic groups admitting a minimally irreducible representation of prime degree are the Mathieu groups $M_{11}, M_{12}, M_{24}$ and the Conway groups $\mathrm{Co}_{2}, \mathrm{Co}_{3}$, and the degree is either 11 or 23 . This is incompatible with the condition $r=\left(q^{n}+1\right) /(q+1)$.

(5) $G \simeq P S p(6,2), r=7$

$P S p(6,2)$ has subgroups isomorphic to the symmetric group $\mathbf{S}_{8}$. Since the minimal degree of a non-linear complex representation of $\mathbf{S}_{\mathbf{8}}$ is 7 , it follows that the representation 
of $\operatorname{PSp}(6,2)$ in question is not minimally irreducible.

(6), (7) The sporadic groups

$M_{12}$ in degree 11 is not minimally irreducible, as the restriction to subgroups isomorphic to $P S L(2,11)$ remains irreducible. Likewise, $M_{24}$ in degree 23 has an irreducible restriction to $P S L(2,23)$. The groups $\mathrm{Co}_{2}$ and $\mathrm{Co}_{3}$ in degree 23 are both minimally irreducible, as the only maximal subgroups of order divisible by 23 are isomorphic to $M_{23}$, and the latter group does not have irreducible representations of degree 23.

We summarise our main results in the following

THEOREM 1. A non-soluble subgroup $G$ of $G L(r, \mathbb{C})$, where $r$ is a prime, is minimally irreducible if and only if one of the following cases occurs:

(i) $G \simeq P S L(2, p), r=p \geqslant 11$;

(ii) $G \simeq P S L(2, q), q \neq 5,9, r=(q+1) / 2$;

(iii) $G \simeq P S L(n, q), n \geqslant 2, q>2, r=\left(q^{n}-1\right) /(q-1)$;

(iv) $G \simeq P S U(n, q),(n, q) \neq(3,3), r=\left(q^{n}+1\right) /(q+1)$;

(v) $G \simeq \mathbb{A}_{5}, r=5$;

(vi) $G \simeq P S L(3,2), r=7$;

(vii) $G \simeq M_{11}, r=11$;

(viii) $G \simeq \mathrm{Co}_{2}, \mathrm{Co}_{3}, r=23$.

In cases (iii), (v), (vi) and (vii) $G$ is monomial, whereas in all the other cases $G$ is a primitive subgroup of $G L(r, \mathbb{C})$.

\section{REFERENCES}

[1] R. Brauer, 'Über endliche lineare Gruppen von Primzahlgrad', Math. Ann. 169 (1967), 73-96.

[2] L. Dornhoff, Group representation theory, Part A (M. Dekker Inc., New York, 1971).

[3] J.D. Dixon and A.E. Zalesskii, 'Finite primitive linear groups of prime degree', J. London Math. Soc. (2) 57 (1998), 126-134.

(4] J.D. Dixon and A.E. Zalesskii, 'Monomial complex finite irreducible linear groups of prime degree', (in preparation).

[5] P. Gérardin, 'Weil representations associated to finite fields', J. Algebra 46 (1977), 54-101.

[6] R.E. Howe, 'On the character of Weil's representation', Trans. Amer. Math. Soc. 177 (1973), 287-298.

[7] B. Huppert, Endliche Gruppen I (Springer-Verlag, Berlin, Heidelberg, New York, 1967).

[8] P. Kleidman and M. Liebeck, The subgroup structure of the finite classical groups, London Math. Soc. Lecture Notes 129 (Cambridge University Press, Cambridge, 1990).

[9] V. Landazuri and G.M. Seitz, 'On the minimal degrees of projective representations of the finite Chevalley groups', J. Algebra 32 (1974), 418-443. 
[10] V.P. Platonov, 'Finiteness of minimal irreducible linear groups', (Russian), Vesc. Acad. Navuk BSSR Ser. Fiz-Mat. Navuk 5 (1975), 96-97.

[11] L. Rédei, 'Das "schiefe Produkt" in der Gruppentheorie mit Anwendung auf die endlichen nichtkommutativen Gruppen mit lauter kommutativen echten Untergruppen und die Ordnungszahlen, zu denen nur kommutative Gruppen gehören', Comment Math. Helv. 20 (1947), 225-264.

[12] G.M. Seitz, 'Some representations of classical groups', J. London Math. Soc. (2) 10 (1975), 115-120.

[13] D.A. Suprunenko, Matrix groups, Transl. Math. Monographs, (translated from the 1972 Russian edition) (Amererican Math. Society, Providence, 1976).

[14] D.A. Suprunenko, 'Minimal irreducible solvable linear groups of prime degree', Soviet Math. Dokl. 13 (1972), 979-981.

[15] D.A. Suprunenko, 'Minimal irreducible solvable linear groups of prime degree', (in Russian), Trudy Moskov. Mat. Obshch. 29 (1973), 223-234.

[16] Pham Huu Tiep and A.E. Zalesskii, 'Some characterizations of the Weil representations of the symplectic and unitary groups', J. Algebra 192 (1997), 130-165.

[17] H.N. Ward, 'Representations of symplectic groups,', J. Algebra 20 (1972), 182-195.

[18] A.E. Zalesskii and I.D. Suprunenko, 'Permutation representations and a fragment of the decomposition matrix of symplectic and special linear groups over a finite field', Siberion Mat. J. 31 (1990), 744-755.

Dipartimento di Matematica e Applicazioni

Università di Milano-Bicocca

via Bicocca degli Arcimboldi 8

I-20126 Milano

Italy

e-mail: daflavolta@matapp.unimib.it

dimartino@matapp.unimib.it 